\title{
Experimental Assessment of the Influence of Interface Geometries on Structural Dynamic Response
}

\author{
T. Dossogne ${ }^{1}$, T.W. Jerome ${ }^{2,5}$, D.P.T. Lancereau ${ }^{3}$, S.A. Smith ${ }^{* 4,5}$, M.R.W. Brake ${ }^{5,6}$, B. Pacini ${ }^{5}$, P. \\ Reu $\AA^{7}$ and C.W. Schwingshackl ${ }^{8}$ \\ ${ }^{1}$ University of Liège, 9, Allée de la Découverte B52/3, 4000 Liège, Belgium \\ ${ }^{2}$ Graduate Program in Acoustics, The Pennsylvania State University, State College, PA 16801, \\ USA \\ ${ }^{3}$ Cranfield University, Shrivenham, Swindon, SN6 8LA, United Kingdom \\ ${ }^{4}$ University of Maryland, Baltimore County, 1000 Hilltop Circle, Baltimore, MD 21250, USA \\ ${ }^{5}$ Sandia National Laboratories ${ }^{\dagger}$, PO Box 5800, Albuquerque, NM 87185, USA \\ ${ }^{6}$ William Marsh Rice University, Houston TX 77005, USA \\ ${ }^{7}$ Daimler AG, 70546 Stuttgart, Germany \\ ${ }^{8}$ Imperial College London, London SW7 2AZ, United Kingdom
}

\begin{abstract}
Jointed interfaces are sources of the greatest amount of uncertainty in the dynamics of a structural assembly. In practice, jointed connections introduce nonlinearity into a system, which is often manifested as a softening response in frequency response, exhibiting amplitude dependent damping and stiffness. Additionally, standard joints are highly susceptible to unrepeatability and variability that make meaningful prediction of the performance of a system prohibitively difficult. This high degree of uncertainty in joint structure predictions is partly due to the physical design of the interface. This paper experimentally assesses the influence of the interface geometry on both the nonlinear and uncertain aspects of jointed connections. The considered structure is the Brake-Reuß beam, which possesses a lap joint with three bolted connections, and can exhibit several different interface configurations. Five configurations with different contact areas are tested, identified, and compared, namely joints with complete contact in the interface, contact only under the pressure cones, contact under an area twice that of the pressure cones, contact only away from the pressure cones and Hertzian contact. The contact only under the pressure cone and Hertzian contact are found to behave linearly in the range of excitation used in this work. The contact area twice that of the pressure cone behaves between the complete contact and contact only under the pressure cone cases.
\end{abstract}

Keywords: Bolted joints; Nonlinear vibrations; Experimental setup; Repeatability test; Lap joint; Pressure cones; Hertzian contact; Micro-slip

\section{Introduction}

Assemblies of systems that are bolted together are prevalent in many engineering applications. The ability to model and predict bolted joint behavior is of great interest, specifically for applications of high consequence, such as in defense, aerospace, and automotive engineering sectors. Such modeling and prediction of the response of bolted structures is a challenging engineering problem for several reasons, including:

- The frictional interactions within the interface of a jointed structure are not well understood, and existing models poorly capture both macroslip and microslip effects [1], unless heurculain efforts are made [2];

- The frictional characteristics of a jointed structure change over time as wear is accumulated [3-8]; and,

- The extent and evolution of the contact patch during dynamic excitation is unknown [9].

*Corresponding author: sasmit@sandia.gov

${ }^{\dagger}$ Sandia National Laboratories is a multi-mission laboratory managed and operated by Sandia Corporation, a wholly owned subsidiary of Lockheed Martin Corporation, for the U.S. Department of Energy's National Nuclear Security Administration under contract DEAC04-94AL85000. 
Another set of problems originates in that structures that are of the same geometric dimensions, material, and assembly process are highly variable in their behavior, exhibiting low repeatability, particularly at modal resonance. If the variability in repeatability due to the material, geometrical, and assembly constraints of the system could be minimized or understood, then models and designers would have some increased measure of predictability of the behavior of systems with bolted joints.

This research endeavors to characterize the important aspects of a system with a bolted joint in terms of repeatability, impact force, and geometric variation via interface area. Analysis of the data is performed with multiple methods to address the inherent and procedural variability of the tested systems. It is expected that some interface areas will feature more variability than others, highlighting information which may be useful to design high-performance structures and create contact models.

\section{Interface design}

In order to assess the effects of interface geometry on the dynamics of a bolted structure, four perturbations of the Brake-Reuß beam [10,11] were designed. The Brake-Reuß beam (BRB) (Fig. 1), is a $72 \mathrm{~cm}$ long beam that is divided into two halves joined by a three-bolt lap joint. The nominal system has a flat interface, as shown in Figure 2(a). This system, however, has exhibited high variability and low repeatability [11-13], and it is hypothesized that this is due to the non-uniform contact pressure across the interface. The four perturbations of this design address two different notions from recent research. First, from the solid mechanics perspective, a traditional lap joint, which is a form of complete contact, is one of the worst designs possible due to the many negative qualities. Complete contacts are characterized by having a sharp edge in their profile, which results in the contact area being independent of the normal load. Additionally, the sharp edge often gives rise to singularities in the stress distributions [14]. For incomplete contact, such as Hertzian contact or contact between two cylinders, the contact area is a function of the applied normal load, and the contact pressure smoothly tends to zero as the contact edge is approached [15]. This notion led to the first BRB perturbation where a Hertzian interface was designed (Figure 2(b)), which consists of four cylindrical bumps that are designed to not yield under the nominal bolt torques used in experiments. Recent research has indicated that most of the dissipation within a jointed interface occurs away from the pressure cones of the bolts [16-20]. Thus, the second perturbation (Figure 2(c), hereafter referred to as the small pad or PDS) was designed to only have contact directly under the pressure cones of the bolts. To explore the transition between the small pad interface and the nominal, flat interface, a large pad (PDL, Figure 2(d)) was designed as a third perturbation. Additionally, the inverse of the PDL (RPD) was designed to see if this significantly exacerbated the dynamics of the system in terms of more variability and less repeatability, and is shown in Figure 2(e).

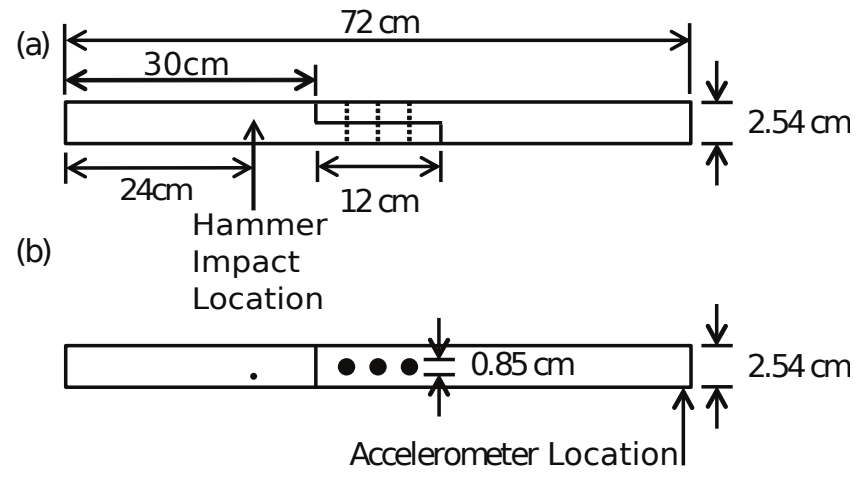

Figure 1: The schematic of the Brake-Reuß beam.

\section{Experimental Setup}

To investigate the effects of the the interface geometry on the response of the beam, impact tests were performed. The beam was assembled using 5/16" - 24 grade- 8 steel bolts. To perform the tests, the beam was suspended on 1.7 $\mathrm{m}$ of bungee that was looped over an $\mathrm{x}$-brace structure, crossed to help prevent beam rolling, and looped around the beam using fishing line. The supports were located approximately $5 \mathrm{~cm}$ in from the edges of the beam. The beam was then impacted using a PCB 086C01 Modal Impact Hammer, at the location shown in Figure 1b. The beam was 


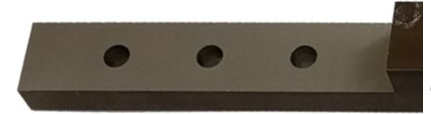

(a)

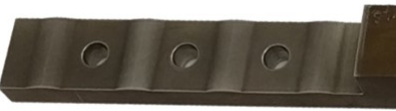

(b)

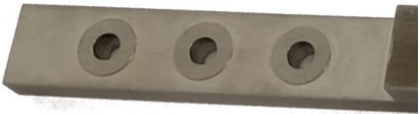

(c)

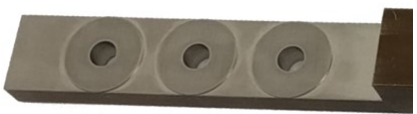

(d)

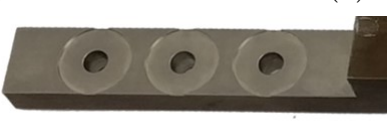

(e)

Figure 2: Interface Designs: (a) Brake-Rueß - BRB, (b) Hertzian Contact - HRZ, (c) Small Pad - PDS, (d) Large Pad PDL, (e) Reverse Pad - RPD.

assembled using a torque of $20 \mathrm{Nm}$, which is lower than the recommend $36.6 \mathrm{Nm}$ for the bolts used. This torque level was selected to exacerbate the nonlinear effects in lower input levels. Three different impact levels were selected, 60 N $210 \mathrm{~N}$ and $340 \mathrm{~N}$, and each level was repeated five times. These force levels were chosen based on the experience from previous testing conducted on the BRB [21]. The response of the system was sampled at $6400 \mathrm{~Hz}$ with a $10 \mathrm{~s}$ recording time and $0.1 \mathrm{~s}$ pre-trigger. The response was measured for $10 \mathrm{~s}$ because the BRB rings for approximately $5 \mathrm{~s}$ and a fine frequency resolution was desired.

To assess the repeatability of the interfaces, the beam is disassembled and reassembled three times. The following procedure was used to increase the likelihood that the only change in the system is the contacts between the microscale disparities. The two parts of the beam were clamped between a uniform metal bar and two shorter beams with the plane of the interface perpendicular to the ground, as shown in Figure 3. Due to the manufacturing process a gap is present at the edge of the beam halves in the interface, which can be seen in Figure 1a. This gap can separate or close depending on the seating of the beam halves. To keep this gap constant a business card is used. The bolts are then tightened, in the order shown in Figure 4, to $70 \%$ of the desired torque level and then to the full level.

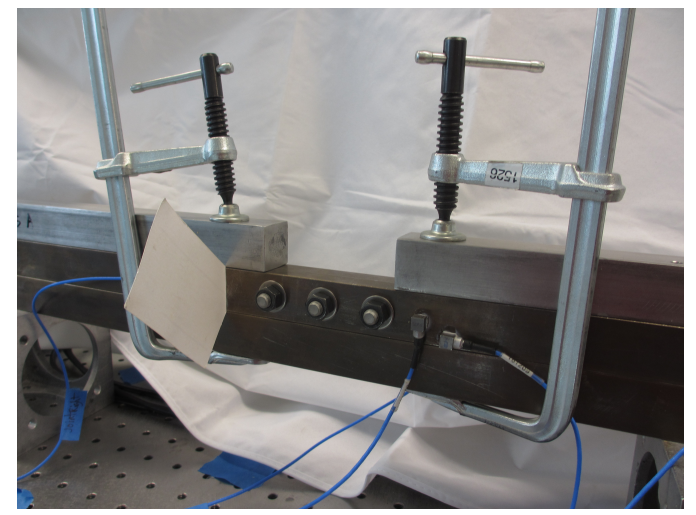

Figure 3: Beam clamped for bolt tightening

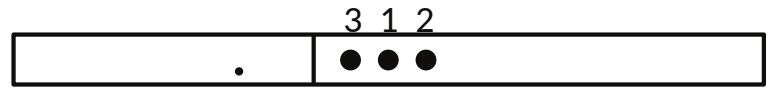

Figure 4: Bolt tightening for all experiments.

\section{Results and Discussion}

\section{Pressure Film}

Before any impact tests were performed, the contact area of the different interfaces were investigated using pressure film. The beam was assembled per the process outlined in the previous section except Fujifilm Prescale medium pressure film was placed in the interface in order to measure the static contact area. The bolts were then torqued 
and held for two minutes. The pressure film results are shown in Figure 5. Note that the bottom image in all of the figures is a digitized version (done by Sensor Products INC.) of the film.

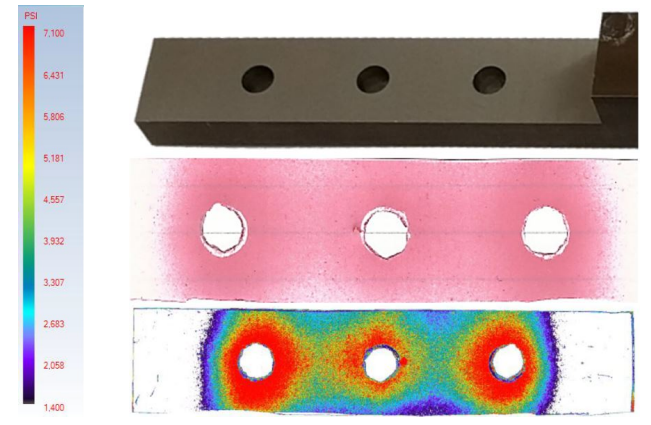

(a)

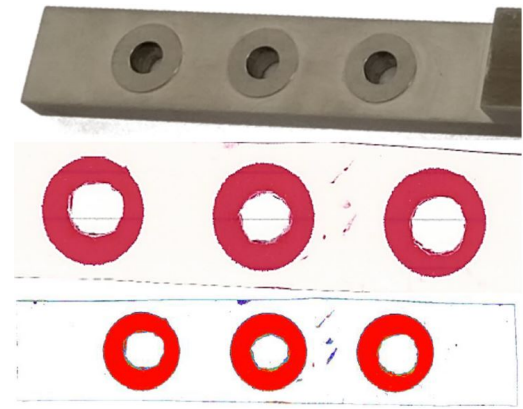

(c)

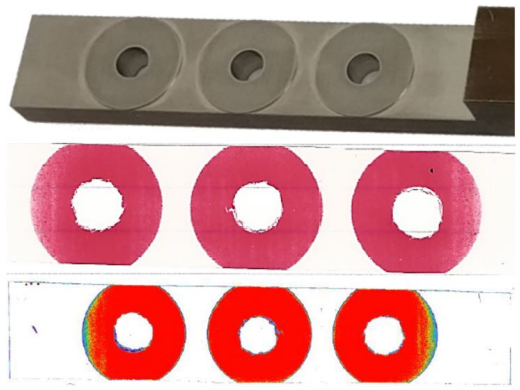

(d)

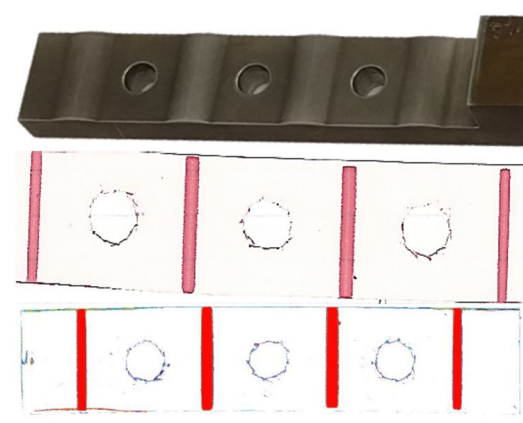

(b)

Figure 5: Pressure film of (a) BRB, (b) HRZ, (c) PDS, (d) PDL, and (e) RPD under $20 \mathrm{Nm}$ bolt torque.

The results of the pressure films of the BRB and HRZ match with the results in [2,22] and Figure 6, respectively. The results for PDS, PDL and RPD also are expected. When the pad is smaller than the pressure cone of the bolts, the resulting contact pressure at the interface is a nearly uniform distribution. Conversely, with the larger pad the pressure distribution is observed to deviate from a uniform distribution near the edges of the outer pads, similar to the BRB. With the reversed pads, a trend similar to the large pad is observed: between the bolts acts like the HRZ, but towards the outer periphery of the interface a significant gradient in the contact pressure is observed.

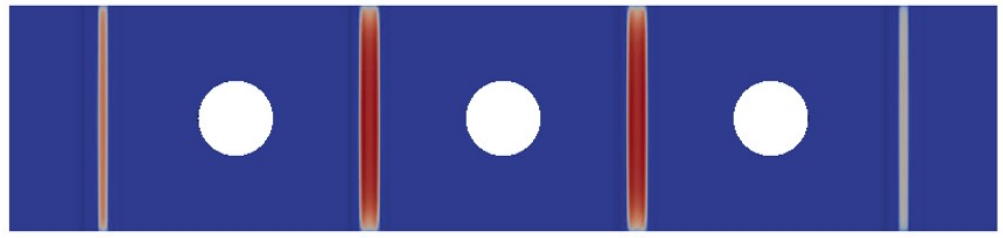

Figure 6: Contact force from finite element simulation of the Hertzian beam (courtesy of Robert Flicek).

Two types of interfaces can be observed in the pressure films: interfaces with sharp edges in the measured contact pressures and interfaces with large gradients in the measured contact pressures. The PDS and HRZ have what appear to be sharp edges, because the contact pressure in these interfaces saturated the pressure film. The BRB and RPD have contact pressures which fade to below $10 \mathrm{MPa}$ at the edges of the interfaces. The PDL is between the two types of trends with pressure near the center of the interface being mostly saturated like the PDS and HRZ, and with the outer edges fading like the BRB and RPD. It is expected that the different interfaces will have significant impact on the nonlinear response of the beam.

Note that for the HRZ and PDS perturbations, the pressure film became saturated. While high-level comparative conclusions could be drawn, these pressure films do not constitute an exhaustive measurement or analysis of the contact pressure distributions for the various BRBs. For a complete understanding of the contact pressure distributions, different films would need to be employed; a less sensitive pressure film should be used where the film becomes saturated and a more sensitive film in the areas where the pressure drops below $10 \mathrm{MPa}$. 


\section{Impact Hammer Testing Analysis}

Following the measurement of the contact pressures, the hammer impact testing was conducted. The results of the impact testing were analyzed using the Hilbert transform [23], the Direct Fitting method [19,20], and the short-time Fourier Transform (STFT) [24]. The nonlinear decay of the first bending mode of each BRB perturbation is compared in Figure 7.
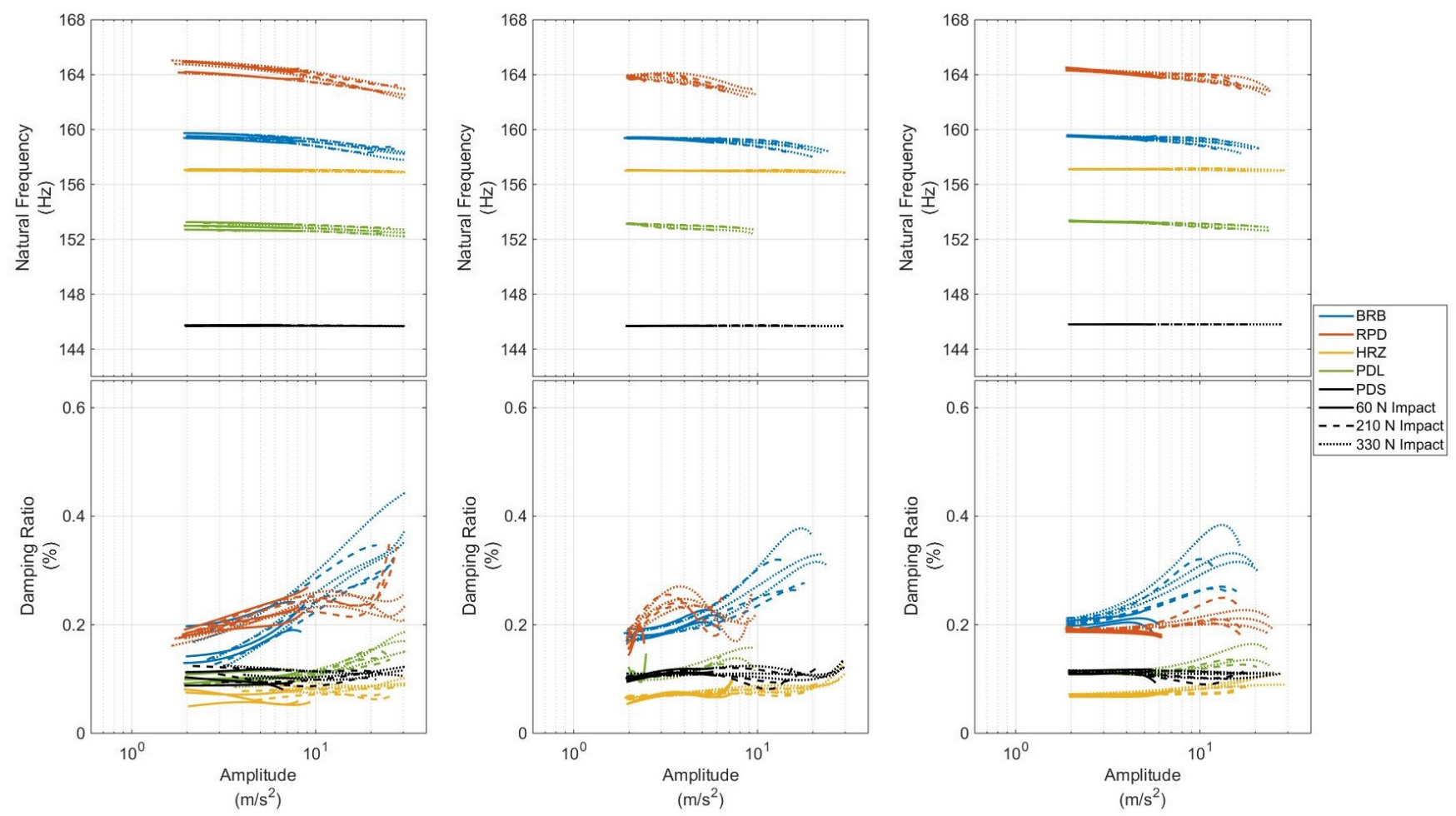

Figure 7: Comparison of the different interfaces under $20 \mathrm{Nm}$ torque; using (left column) Hilbert Transform, (center column) Direct Fitting, and (right column) STFT.

The RPD has the highest frequency, which could be from the mass at the interface being lower than the BRB, but the contact stiffness was not drastically changed (as opposed to the HRZ or PDS in which the contact stiffness of the interface is significantly changed while the mass is also reduced significantly). The HRZ and BRB have similar frequencies, which means that even though the contact stiffness of the HRZ maybe lower than the BRB, the removal of the material to create the HRZ interface is great enough to keep the contact stiffness-mass ratio similar. The PDS has the lowest frequency of all interfaces, and it is the most linear of all of the interfaces. Both the PDS and the HRZ interfaces have the lowest damping ratios, which indicates that redesigning the the interface to have higher contact pressure reduces micro-slip, which is a large source of nonlinearity in joints. The PDL, as anticipated, shows a weaker nonlinearity than that observed in the BRB, but a stronger nonlinearity than observed in the PDS.

To assess the repeatability and variability of these systems a procedure was adopted where the dynamics of the beam were measured, the system was then disassembled, the interface was re-seated, the dynamics of the system were measured again, and the process was repeated. The frequency of the different interfaces is fairly repeatable, while the damping has large variations. The damping of systems more susceptible to changes in micro-structure (due the engagement of the disparities changing with each re-seat of the interface) of the interfaces than the frequency, which is indicated in 7 by the width of the measured damping responses compared to the frequency.

\section{Discussion}

While there is variability in the behavior of the nonlinearities for each BRB perturbation, distinctions can be made. The Brake-Reuß beam variants designed with large amounts of contact area farther from the bolt hole were less repeatable and exhibited large variability in the observed nonlinear behavior. Other observations made include:

- Interfaces with high pressure concentrations (PDS \& HRZ) behaved linearly due to slip not occurring at the excitation levels achieved in this work; 
- Interfaces that had more gradual pressure gradients (BRB, RPD, \& PDL) behaved more nonlinearly due to micro-slip occuring in the transition zones; and,

- Damping exhibited a higher amount of variability than frequency due to the damping being more susceptible to changes in disparity contact.

An interesting result observed is that the contact of the PDL, seen in Figure 5d, has characteristics of the BRB and PDS interfaces; the center pad of the PDL was saturated like the PDS, whereas a gradual pressure change was observed on the outer pads similar to the gradient of the BRB. This is mirrored in the frequency and damping variations of the PDL, seen in Figure 7, were it exibits similar nonlinear trends as the BRB at higher excitation levels, and linear trends as the PDS at lower levels. These results suggest that gradual pressure gradients and free edges are a large source of nonlinear effects in joints, while an interface in complete contact behaves linearly.

The three different techniques (Hilbert Transform, Direct Fitting, and STFT) used to analyze the impact response, have similar results for frequency and damping. Near the end of the signal the Direct Fitting and STFT start to have difficulties fitting the data for extracting damping. The main differences between the three techniques is how the data is extracted; the Hilbert Transform and Direct Fitting need the response to be filtered, and the damping is extracted by regression fitting (Hilbert Transform), exponential fitting (Direct Fitting), and the amplitude of the max Fourier coefficient (STFT).

\section{Conclusion}

The study of different interface geometries has provided insight into the possibility of designing interfaces that behave as expected, as well as having linear properties. The pressure film has proven to be a useful tool in this study to help provide insights into the dynamic behavior of the structural joint. It is found that gradual pressure gradients and free edges are a large source of nonlinear effects in joints, while an interface in complete contact behaves linearly. The two interfaces with abrupt change of contact pressure (HRZ and PDS), represent systems that have linear behavior. The three interfaces with a fading pressure distribution display nonlinear behavior. These results corroborate the hypotheses that micro-slip at the edge of the interface contact is the origin of nonlinear damping in the system. This study demonstrates that the design of the joint clearly influences the dynamic behavior of the structure.

\section{Acknowledgments}

The authors would like to thank Randall Mayes from Sandia National Laboratories for his input on updating the test setup; as well as Jeffrey Stark from Sensor Products INC. for his help with digitizing the pressure films.

\section{References}

[1] D. J. Segalman, D. L. Gregory, M. J. Starr, B. R. Resor, M. D. Jew, J. P. Lauffer, and N. M. Ames, "Handbook on dynamics of jointed structures," Sandia National Laboratories, Albuquerque, NM, 2009.

[2] R. M. Lacayo, L. Pesaresi, D. Fochler, J. Groß, M. R. W. Brake, and C. W. Schwingshackl, "Instantaneous frequency and damping from transient ring-down data," in IMAC XXXV A Conference and Exposition on Structural Dynamics, (Garden Grove, CA), February 2017.

[3] M. Müller and G. Ostermeyer, "A cellular automaton model to describe the three-dimensional friction and wear mechanism of brake systems," Wear, vol. 263, no. 7, pp. 1175-1188, 2007.

[4] G. Ostermeyer and M. Müller, "Dynamic interaction of friction and surface topography in brake systems," Tribology International, vol. 39, no. 5, pp. 370-380, 2006.

[5] M. Müller and G. Ostermeyer, "Cellular automata method for macroscopic surface and friction dynamics in brake systems," Tribology International, vol. 40, no. 6, pp. 942-952, 2007.

[6] K. Bode and G.-P. Ostermeyer, "A comprehensive approach for the simulation of heat and heat-induced phenomena in friction materials," Wear, vol. 311, no. 1, pp. 47-56, 2014.

[7] D. Mulvihill, M. Kartal, D. Nowell, and D. Hills, "An elastic-plastic asperity interaction model for sliding friction," Tribology international, vol. 44, no. 12, pp. 1679-1694, 2011. 
[8] C. Schwingshackl, E. Petrov, and D. Ewins, "Measured and estimated friction interface parameters in a nonlinear dynamic analysis," Mechanical Systems and Signal Processing, vol. 28, pp. 574-584, 2012.

[9] M. R. W. Brake, J. G. Stark, S. A. Smith, T. W. J. D. P. T. Lancereau, and T. Dossogne, "In situ measurements of contact pressure for jointed interfaces during dynamic loading experiments," in IMAC XXXV A Conference and Exposition on Structural Dynamics, (Garden Grove, CA), February 2017.

[10] M. R. W. Brake, ed., The Mechanics of Jointed Structures. Springer, 2016.

[11] M. R. Brake, P. Reuß, D. J. Segalman, and L. Gaul, "Variability and repeatability of jointed structures with frictional interfaces," (Orlando, FL), IMAC XXXII A Conference and Exposition on Structural Dynamics, February 2014.

[12] S. A. Smith, J. C. Bilbao-Ludena, S. Catalfamo, M. R. W. Brake, P. Reuß, and C. W. Schwingshackl, "The effects of boundary conditions, measurement techniques, and excitation type on measurements of the properties of mechanical joints," in IMAC XXXII A Conference and Exposition on Structural Dynamics, (Orlando, FL), February 2015.

[13] S. Catalfamo, S. A. Smith, F. Morlock, M. R. W. Brake, P. Reuß, and C. W. Schwingshackl, "Effects of experimental methods on the measurement of a nonlinear structure," in IMAC XXXIII A Conference and Exposition on Structural Dynamics, (Orlando, FL), February 2016.

[14] J. R. Barber, A. Klarbring, and M. Ciavarella, "Shakedown in frictional contact problems for the continuum," Comptes Rendus Mecanique, vol. 336, pp. 34-41, 2008.

[15] C. Churchman and D. Hills, "General results for complete contacts subject to oscillatory shear," Journal of the Mechanics and Physics of Solids, vol. 54, no. 6, pp. 1186-1205, 2006.

[16] H. G. D. Goyder, P. Ind, and D. Brown, "Measurement of damping in bolted joints," in ASME International Design Engineering Technical Conferences IDETC/CIE, (Chicago, IL), August 2012.

[17] H. G. D. Goyder, P. Ind, and D. Brown, "Measurement of damping due to bolted joints," in ASME International Design Engineering Technical Conferences IDETC/CIE, (Portland, OR), August 2013.

[18] H. G. D. Goyder, P. Ind, and D. Brown, "Measurement of damping in a chain of bolted joints," in $A S M E$ International Design Engineering Technical Conferences IDETC/CIE, (Buffalo, NY), August 2014.

[19] H. G. D. Goyder, P. Ind, and D. Brown, "Damping in a composite beam with a joined interface," in $A S M E$ International Design Engineering Technical Conferences IDETC/CIE, (Boston, MA), August 2015.

[20] H. G. D. Goyder, D. P. T. Lancereau, P. Ind, and D. Brown, "Friction and damping associated with bolted joints: Results and signal processing," in Proceedings of the ISMA 2016 International Conference on Noise and Vibration Engineering, (Leuven, Belgium), September 2016.

[21] M. S. Bonney, B. A. Robertson, F. Schempp, M. Mignolet, and M. R. Brake, "Experimental determination of frictional interface models," in IMAC XXXIV A Conference and Exposition on Structural Dynamics, (Orlando, FL), February 2016.

[22] R. C. Flicek, K. J. Moore, G. M. Castelluccio, C. Hammetter, and M. R. W. Brake, "Stress waves propagating through jointed connections," in IMAC XXXIV A Conference and Exposition on Structural Dynamics, (Orlando, FL), February 2016.

[23] M. W. Sracic, M. S. Allen, and H. Sumali, Identifying the Modal Properties of Nonlinear Structures Using Measured Free Response Time Histories from a Scanning Laser Doppler Vibrometer, pp. 269-286. New York, NY: Springer New York, 2012.

[24] R. J. Kuether and M. R. W. Brake, "Instantaneous frequency and damping from transient ring-down data," in IMAC XXXIV A Conference and Exposition on Structural Dynamics, (Orlando, FL), February 2016. 